\title{
The impact of accurate mass measurements using quadrupole/time-of-flight mass spectrometry on the characterisation and screening of drug metabolites
}

\author{
G. Hopfgartner ${ }^{1 *}$ and F. Vilbois ${ }^{2}$ \\ ${ }^{1}$ F. Hoffmann-La Roche Ltd. Pharmaceuticals Division, Non Clinical Development-Drug Safety, Bld. 68/142, CH-4070 Basel, Switzerland \\ ${ }^{2}$ Serono Pharmaceutical Research Institute, Department of Biotechnology, CH-1228 Plan-les-Ouates, Geneva, Switzerland
}

\begin{abstract}
Atmospheric pressure ionisation quadrupole/ time-of-flight mass spectrometry was used to perform accurate mass measurements on the product ion fragments of moclobemide and remikiren. The low energy collision induced dissociation fragmentation pathways of the two compounds could be established using accurate mass, MS $^{n}$ data and MS interpretation software (Mass Frontier). An atypical fragmentation corresponding to the loss of neutral dimethylvinyl could be clearly identified. The knowledge gained from these experiments was used to demonstrate the application of data dependent experiments on a triple quadrupole instrument for the screening of metabolites. Data dependent experiments were performed using either Q3 single scan or precursor ion scan mode as the survey scan, and product ion scan as the dependent scan.
\end{abstract}

\section{Introduction}

The study of drug candidate biotransformation during the drug discovery and drug development stages requires the structural characterisation as well as the screening of in vitro or in vivo generated metabolites. Acceleration of drug discovery timelines are calling for improved approaches to support this type of work. Liquid chromatography combined with mass spectrometric detection (LC-MS) and atmospheric pressure ionisation (API) is particularly efficient for analysing very low amounts of pharmaceutical compounds in complex matrices such as bile, plasma or urine [1]. With LC-MS, retention time as well as molecular weight information can be obtained. Phase I metabolism will often result in small changes in the molecular weight of the candidate drug such as, the addition of an oxygen atom or the loss of an alkyl moiety. These changes are very common. However, 
one would like to know as fast as possible where they are occurring on the molecule, which is a challenging task.

API ionisation such as ion spray or APCI generate mainly protonated or deprotonated molecules in the positive or negative mode, respectively [2,3]. These ionisation modes are particularly suitable for the application of tandem mass spectrometry [4]. Triple quadrupole (QqQ) and ion trap mass spectrometers operated under various scanning modes such as product ion, precursor ion and neutral loss are largely used to identify and characterise metabolites [5].

To fully benefit from these MS/MS techniques it is very important to have a clear understanding of the fragmentation mechanism generated by collision induced dissociation (CID). Unfortunately, this process is often very complicated [6]. Ion fragments and intensities cannot be predicted. Typically, product ion spectra are obtained with a triple quadrupole instrument, which operates at unit mass resolution. Classically, the product ion spectrum of the parent drug is used as a structural template for the identification of the structure of the metabolites. However, different fragmentation pathways can lead to fragments with the same nominal mass (isobaric ions). It is known that these pathways are very sensitive to small structural changes [7]. Therefore, a mass shift of $16 \mathrm{Da}$ for a supposed fragment or for an oxidative metabolite can easily be misinterpreted, leading to an incorrect structural proposal. Ion trap mass analysers generate $\mathrm{MS}^{\mathrm{n}}$ in time CID spectra at medium resolution. This is particularly useful to follow a fragmentation cascade. Recently, the combination of the quadrupole mass analyser with a time-of-flight mass analyser (QqTOF) has been described [8,9]. QqTOF is particularly powerful to obtain product ion spectra at medium resolution with the possibility of accurate mass measurement of the fragments. This opens a new dimension in the rational interpretation of CID spectra using elemental composition in combination with MS interpretation software. The aim of the present study is to illustrate with two examples; moclobemide and remikiren, the benefit of accurate mass measurement in the tandem MS mode and its impact in metabolite screening using also triple quadrupole mass spectrometers and data dependent experiments. Other approaches such as the use of isotopic shift were not considered in this paper.

\section{Experimental}

\section{Tandem MS accurate mass measurements with QqTOF}

Accurate mass measurements were performed using pneumatically-assisted electrospray by infusion $(0.1-1 \mathrm{ng} / \mu \mathrm{l}$ $50 / 50$ in $\mathrm{H}_{2} \mathrm{O} / \mathrm{MeOH} 1 \% \mathrm{CH}_{3} \mathrm{COOH}$ ) on a Micromass Q-TOF operating in the positive mode. Instrument calibration was achieved by infusion of a solution of quaternary ammonium halides in the mass range $\mathrm{m} / \mathrm{z} 50$ to 1000 . The duty cycle was set from 0.1 to $2.4 \mathrm{sec}$. The samples were introduced by infusion at a flow rate of $5 \mu \mathrm{l} / \mathrm{min}$. MS/MS experiments were performed with a quadrupole resolution of $2 \mathrm{Da}$. The collision gas (argon) was set $5.8 \times 10^{-5} \mathrm{mbar}$. Ions were pulsed into the flight tube at a potential of $7200 \mathrm{~V}$ and detected with a multi-channel plate detector set at $2800 \mathrm{~V}$. Control data acquisition and data reduction were carried out by Masslynx version 3.2.

\section{Ion trap spectra}

$\mathrm{MS}^{\mathrm{n}}$ spectra were obtained on a classic LCQ ion trap (Finnigan) by infusion of reference solutions (0.1-1 ng/ $\mu \mathrm{l}$ in $\left.50 / 50 \mathrm{H}_{2} \mathrm{O} / \mathrm{MeOH} 1 \% \mathrm{CH}_{3} \mathrm{COOH}\right)$ at $5 \mu \mathrm{l} / \mathrm{min}$ using pneumatically-assisted electrospray in the positive mode. The temperature of the capillary was set at $200{ }^{\circ} \mathrm{C}$. Control data acquisition and data reduction were carried out by Excalibur version 1.0 .

\section{LC-MS analysis of moclobemide}

For the analysis of $10 \mu \mathrm{M}$ moclobemide incubated $(24 \mathrm{~h})$ in rat hepatocytes two LC10AD pumps (Shimadzu) were used to provide a high-pressure gradient for the analytical column (Inertsil ODS-3 $150 \times 2.1 \mathrm{~mm}$ ) using a dynamic mixer $(75 \mu \mathrm{l})$. The sample solution was injected $(20 \mu \mathrm{l})$ by means of a Gilson 234 autosampler. The gradient started 2 min after injection with $0 \% \mathrm{~B}$, and the proportion of $\mathrm{B}$ was increased to $60 \%$ within $10 \mathrm{~min}$ at a flow rate of $0.25 \mathrm{ml} / \mathrm{min}$ and hold for 5 minutes. Solvent A consisted of a mixture $5 \mathrm{mM}$ ammonium acetate with $1 \%$ acetic acid, whereas solvent B consisted of a mixture methanol-acetonitrile-acetic acid in the ratio 50/50/1 (v/v/v). Mass spectrometric detection was performed on a triple quadrupole API 3000 (PE Sciex) with Analyst 1.1 and DDE add-in in positive mode using a turbo ion spray interface. Nitrogen was used as the collision gas and the collision energy was set at $30 \mathrm{eV}$.

\section{LC-MS analysis of remikiren}

The HPLC hardware was similar to that used for the analysis of moclobemide using a X-Terra MS C18 $2.5 \mathrm{~m}$ analytical column (Waters). After injection the gradient started with $30 \% \mathrm{~B}$, and the proportion of $\mathrm{B}$ was increased to $70 \%$ within $10 \mathrm{~min}$ at a flow rate of $0.25 \mathrm{ml} / \mathrm{min}$ and hold for 5 minutes. Solvent A consisted of $1 \%$ acetic acid, whereas solvent B consisted of a mixture methanol-acetonitrile-acetic acid in the ratio 50:50:1 (v/v/v).

Mass spectrometric detection was performed on a triple quadrupole API 3000 (PE Sciex) with Analyst 1.1 and DDE add-in in positive mode using a turbo ion spray interface. Nitrogen was used as the collision gas and the collision energy was set at $50 \mathrm{eV}$.

\section{Data dependent experiments}

Data Dependent Experiment (DDE) is a procedure used in order to maximise the amount of information that can be obtained in a single LC-MS/MS run [10]. Basically, it combines in the same run two different scan modes in a sequential way such as i) full scan and product ion mode or ii) 
precursor ion mode and product ion mode. The first scan is defined as the survey scan, where the data are rapidly processed in order to determine the ions of interest (candidates). The selection criteria can be: ion intensities, selected $\mathrm{m} / \mathrm{z}$ values or mass ranges. The second scan (dependent scan) is than performed on the candidates using the selection criteria. For the LC-MS analysis of moclobemide the survey scan was a Q3 full scan and the dependent scan a product ion scan. To allow fast switching from single MS to MS/MS the collision gas needs to be on all the time. Therefore single MS spectra can only be recorded with Q3. The selection criteria were to use as candidates either ions with following $\mathrm{m} / \mathrm{z}$ values 241, 243, 269, 283, 285, 299 and 301 , corresponding to the calculated theoretical $[\mathrm{M}+\mathrm{H}]^{+}$ ions of potential metabolites or the most intense ion observed in the $\mathrm{m} / \mathrm{z}$ range $200-500$. For the LC-MS analysis of remikiren the survey scan was a precursor ion scan on the fragment at $\mathrm{m} / \mathrm{z}, 110$ and the dependent scan a product ion scan on the most intense precursor ion in the $\mathrm{m} / \mathrm{z}$ range 400-800. The scan time for all experiments was set to $1 \mathrm{sec}$ and the step to 0.1 amu for both modes.

\section{MS interpretation software}

Mass Frontier 1.0 (Highchem) is a software package for the management an interpretation of mass spectra for small molecules. Fragments and mechanisms can be generated in two ways: electron impact and protonation mode. The software is designed only for neutral and single positively charged molecules.

\section{Results and discussion}

In drug metabolism phase I and phase II reactions are relatively well known. In most cases based on the constraint of the parent drug and the nominal mass obtained by LC-MS on quadrupole instruments, the elemental formula of most metabolites can be easily deduced. However, in some isolated cases accurate mass measurements are necessary. Historically, accurate mass measurements were performed on double focusing magnetic sector instruments using either electron impact or fast atom bombardment ionisation. The measurements were performed at high resolution, typically better than 30000 at $10 \%$ valley with a mass accuracy better than $2 \mathrm{ppm}$. Ion spray ionisation has been successfully interfaced with magnetic sector instruments or Fourier-transform ion cyclotron resonance (FT-ICR) mass spectrometers.

Nowadays, the most common mass analysers used for exact mass measurements are the time-of-flight mass analysers $[11,12]$. Accurate mass measurements can also be performed on quadrupole instruments [13]. However, one major limitation is the possible presence of interfering peaks at the same nominal mass as the metabolite. The resolution of time-of-flight instruments is no longer reported at $10 \%$ valley but as the peak width at $50 \%$ height (FWHM). Compared to a double focusing magnetic sector instrument, where resolutions of 25000-50000 (FWHM) can be achieved, the type of resolution of the TOF has to be considered as medium, typically between 6000 and 10000 (FWHM).

Structural information of molecules can be obtained by collision-induced dissociation in a quadrupole collision cell. However, to fully benefit from this information, an understanding of the fragmentation pathway is required. Single lock mass calibration drift correction on a QqTOF instrument allows achieving accuracies better than $5 \mathrm{ppm}$ on the product ion fragments [7]. It requires either a residual precursor ion or a fragment of known mass.

Using a "basket in a basket" approach based on a multistage accurate mass spectrometric technique $\mathrm{Wu}$ [14] has reported the application to the structure elucidation for a compound obtained by combinatorial synthesis, using a FT-ICR mass spectrometer. Using direct accurate mass measurement, accuracy better than $0.02 \mathrm{ppm}$ is required to define a unique elemental composition.

In drug metabolism studies the structure of the drug candidate is known and it is assumed that reasonable structures can be proposed for any fragment using elemental composition. Ideally, smart software should perform this task automatically, which would allow building knowledge on the mechanism of low energy CID fragmentation.

Accurate mass measurements of small molecules using TOF or QqTOF mass analysers are obtained while performing the simultaneous measurement of the mass of a reference compound and of the ions of interest. The exact mass of this reference is used as a lock mass to correct for the drift of the instrument's calibration. Various factors like instrumentation set-up, isobaric fragments in MS/MS mode originating from the same molecule or interfering compounds, can affect the accuracy of the measurements. It is also difficult to obtain accurate mass measurements during LC-MS analyses. There is a need for alternative methods. Eckers et al. [15] have recently reported the use of separated sprayers for the introduction of sample and reference. Accurate mass measurements can be affected by instrumentation set-up isobaric fragments originating from the same molecule of interfering compounds. Ideally, with more complex molecules one would like to have a resolution better than 25000 (FWHM) and accuracy better than 1 ppm, which currently cannot be achieved on commercial QqTOF instruments.

\section{Analysis of moclobemide}

The CID spectrum at $15 \mathrm{eV}$ of moclobemide (I, Scheme 1) using single mass lock of the residual precursor ion is illustrated in figure 1A. The Mass Frontier software proposes a single structure of the fragment at $\mathrm{m} / \mathrm{z} 182$ with following elemental composition, $\mathrm{C}_{9} \mathrm{H}_{9} \mathrm{CINO}, \mathrm{EM}=182.0373$. The variation between the calculated and the measured mass of the fragment is less than $5 \mathrm{ppm}$. This is in full agreement with the proposed structure. In order to perform single lock mass calibration it is mandatory to use the mass of a known fragment such as the residual precursor ion. However, the 
I $\mathrm{MW}=268.8$<smiles>O=C(NCCN1CCOCC1)c1ccc(Cl)cc1</smiles>

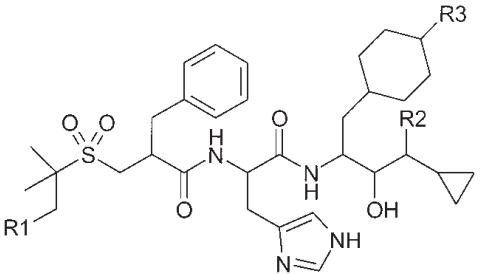

II $\mathrm{R} 1=\mathrm{H}, \mathrm{R} 2=\mathrm{OH}, \mathrm{R} 3=\mathrm{H} \mathrm{MW}=630.9$

III R1 $=\mathrm{H}, \mathrm{R} 2=\mathrm{O}, \mathrm{R} 3=\mathrm{H} \mathrm{MW}=628.9$

IV R1 $=\mathrm{OH}, \mathrm{R} 2=\mathrm{OH}, \mathrm{R} 3=\mathrm{H} \mathrm{MW}=646.8$

$\mathrm{V} R 1=\mathrm{H}, \mathrm{R} 2=\mathrm{OH}, \mathrm{R} 3=\mathrm{OH} \mathrm{MW}=646.8$
Scheme 1. Structures moclobemide (I), remikiren (II) and several metabolites (III-V).

aspect of the product ion spectrum depends on the collision energy. Too little energy will not generate the relevant fragment ions and too much energy will result in the disappearance of the precursor ion as illustrated in figure 1B. One solution is to analyse systematically all samples at two different collision energies and to combine the spectra. This approach was used to measure the accurate masses in spectrum 1B. For the structure of fragment at $\mathrm{m} / \mathrm{z} 139$ Mass Frontier proposes two options (1) and (2) in figure 1B. By using accurate mass measurements it is obvious that only $\mathrm{C}_{7} \mathrm{H}_{4} \mathrm{ClO}$ is a possible fragment even with $15 \mathrm{ppm}$ inaccuracy. Fragment (2) has an inaccuracy of $646 \mathrm{ppm}$ and is also too exotic to exist as a true chemical entity. This illustrates the problems associated with automated CID spectra interpretation to propose potential fragment structures. Two additional limitations remain to be resolved in order to obtain reliable results and thus allow performing high-throughput structural elucidation of CID spectra. The first is continuous instrument calibration. Single point recalibration does not always assure linear calibration over a large mass range of several hundred daltons. The second is isobaric ions. At medium resolution, the mass measurement can be affected by these. $\mathrm{MS}^{\mathrm{n}}$ can be an elegant orthogonal approach to achieve increased accuracy and resolution. For example, in the case of moclobemide the $\mathrm{MS}^{2}$ spectrum of the precursor ion at $\mathrm{m} / \mathrm{z} 269$ shows a strong fragment at $\mathrm{m} / \mathrm{z} 182$ (figure $1 \mathrm{~A})$. Further fragmentation of this ion $\left(\mathrm{MS}^{3}\right)$ generates a strong fragment at $m / z, 139$, indicating that only structure (1) is possible.

The understanding of the CID fragmentation mechanism is important for the structural elucidation of metabolites, but it also allows fast screening of metabolites in biological matrices using non-radiolabelled drugs. Compared to quantitative analysis the LC-MS run cycle time in drug metabolism studies is relatively long in order to achieve good chromatographic separation. An efficient mass spectrometric set-up to perform the LC-MS analysis of metabolites is the use of Data Dependent Experiments, which allows obtaining more structural information in the same run. Typically a single MS survey scan is performed and processed in order to locate mass peaks of interest. Either the most intense ion in the spectrum or an ion from a list is selected in the dependent scan. The survey and dependent scans are repeated throughout the analytical run. DDE experiments using

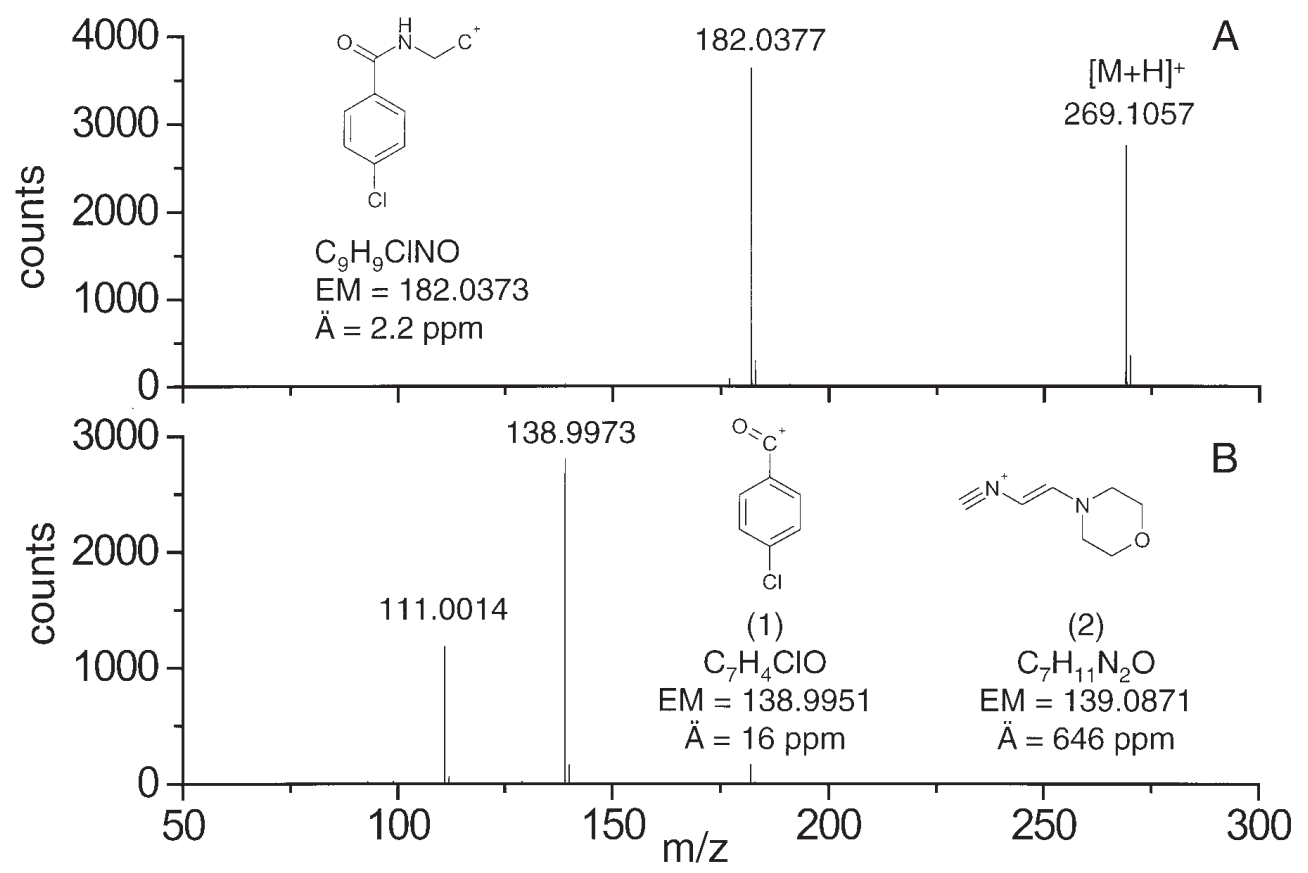

Figure 1. Product ion spectra of moclobemide on a QqTOF A) $15 \mathrm{eV}$ B) $40 \mathrm{eV}$. 


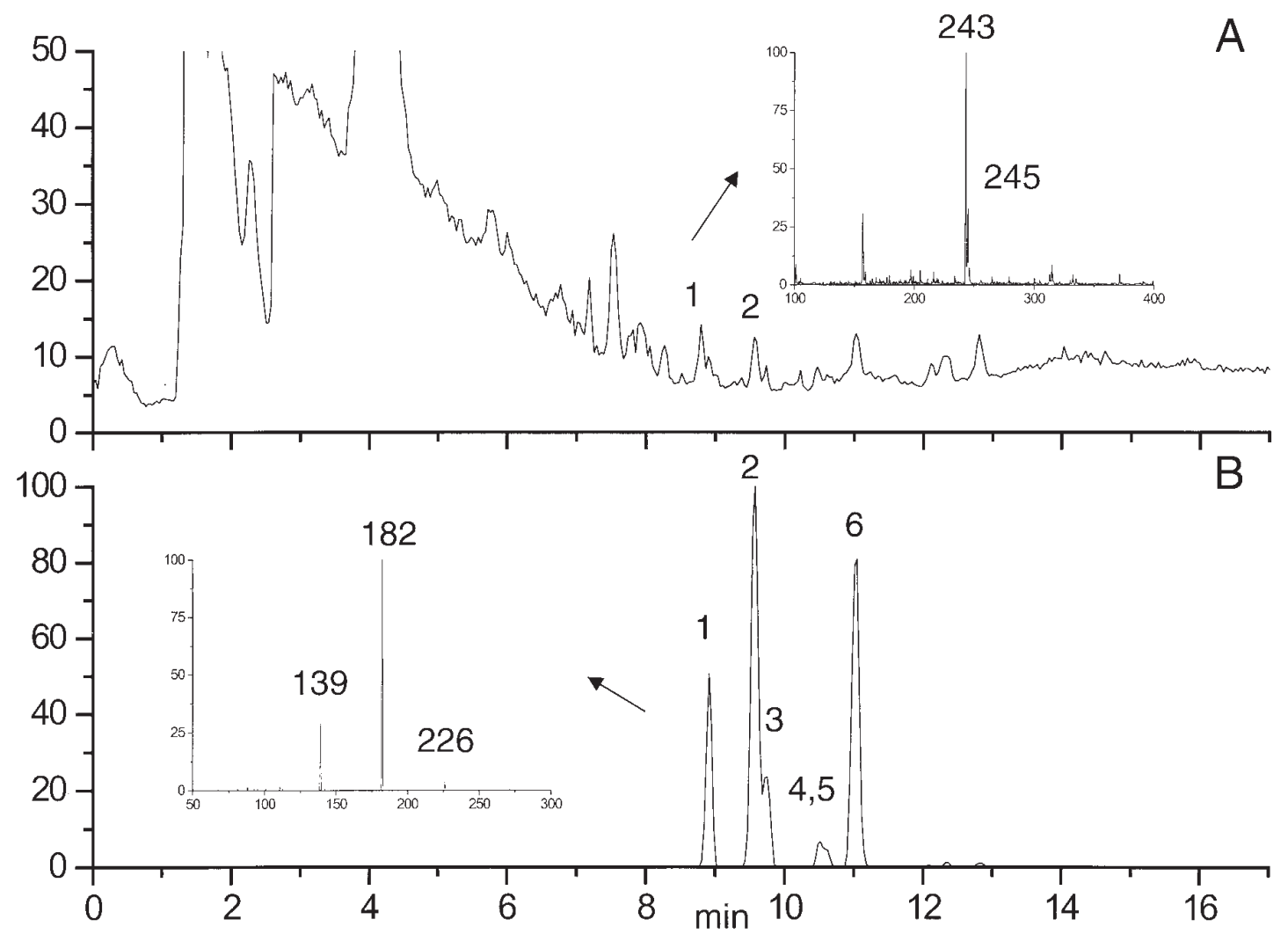

Figure 2. LC-MS/MS DDE experiment on a triple quadrupole of moclobemide incubated with rat hepatocytes A) Q3 TIC trace (survey scan) B) extracted product ion trace at $m / z 182$ (dependent scan) (2) moclobemide.

single MS as survey scan and product ion as dependent scan can be performed on ion trap, QqTOF and QqQ instruments.

Moclobemide belongs to the monoamine oxidase inhibitors of the benzamide type and contains a morpholine moiety. Moclobemide is extensively metabolised [16] and the metabolic pathway includes mainly oxidative attacks on the morpholine moiety. The application of DDE to the analysis of moclobemide incubated in rat hepatocytes is illustrated in figure 2 using a QqQ instrument. Figure 2A shows the LC-MS TIC trace (survey scan), while figure 2B shows the extracted ion chromatogram for $\mathrm{m} / \mathrm{z}, 182$ from the product ion trace (dependent scan). By this way it is possible to identify metabolites of moclobemide very easily with a precursor look like experiment. The basis of this type of experiment is the selection of representative precursor ions. Therefore, an understanding of the fragmentation process is essential to avoid misinterpretation. This approach also requires a list of the predicted masses corresponding to possible metabolic transformations.

\section{Analysis of Remikiren}

Remikiren (II, Scheme 1) is a potent and specific inhibitor of human renin [17] with a molecular weight of $630.9 \mathrm{Da}$.
Remikiren extensively metabolises and compounds III to V represent some of the oxidative metabolites. The low resolution CID spectrum of Remikiren is shown in figure 3A and many fragments are observed. The interpretation of the many fragments in this spectrum is quite difficult. By assumption one could find a sensible structure for each of these fragments, but it could be associated to mass poetry. The fragment at $\mathrm{m} / \mathrm{z}, 613$ is obtained after the loss of water, while the fragments at $m / z 404$ and 376 represent $a$ and $b$ cleavage products of the amide bond as illustrated in scheme 2 . The fragments smaller then $\mathrm{m} / \mathrm{z} 300$ are very difficult to interpret, because several structures are possible. In order to rapidly identify potential metabolites and to find diagnostic ions for the search of non-labelled metabolites an understanding of the fragmentation pathway becomes mandatory. Classically, one would use structural analogues to characterise the fragments, but this approach requires synthetic reference compounds and is quite time-consuming [6]. For the fragment at $\mathrm{m} / \mathrm{z} 282$ three propositions are made by Mass Frontier as illustrated in scheme $3 . \mathrm{MS}^{2}$ and $\mathrm{MS}^{3}$ spectra of remikiren are illustrated in figure $3 \mathrm{~B}$ and $3 \mathrm{C}$. It can be concluded that the ion at $\mathrm{m} / \mathrm{z} 282$ is not generated from the precursor fragment at $\mathrm{m} / \mathrm{z}$ 376. However, an $\mathrm{MS}^{3}$ experiment 631 -> 404 -> (data not shown) generates an abundant ion 


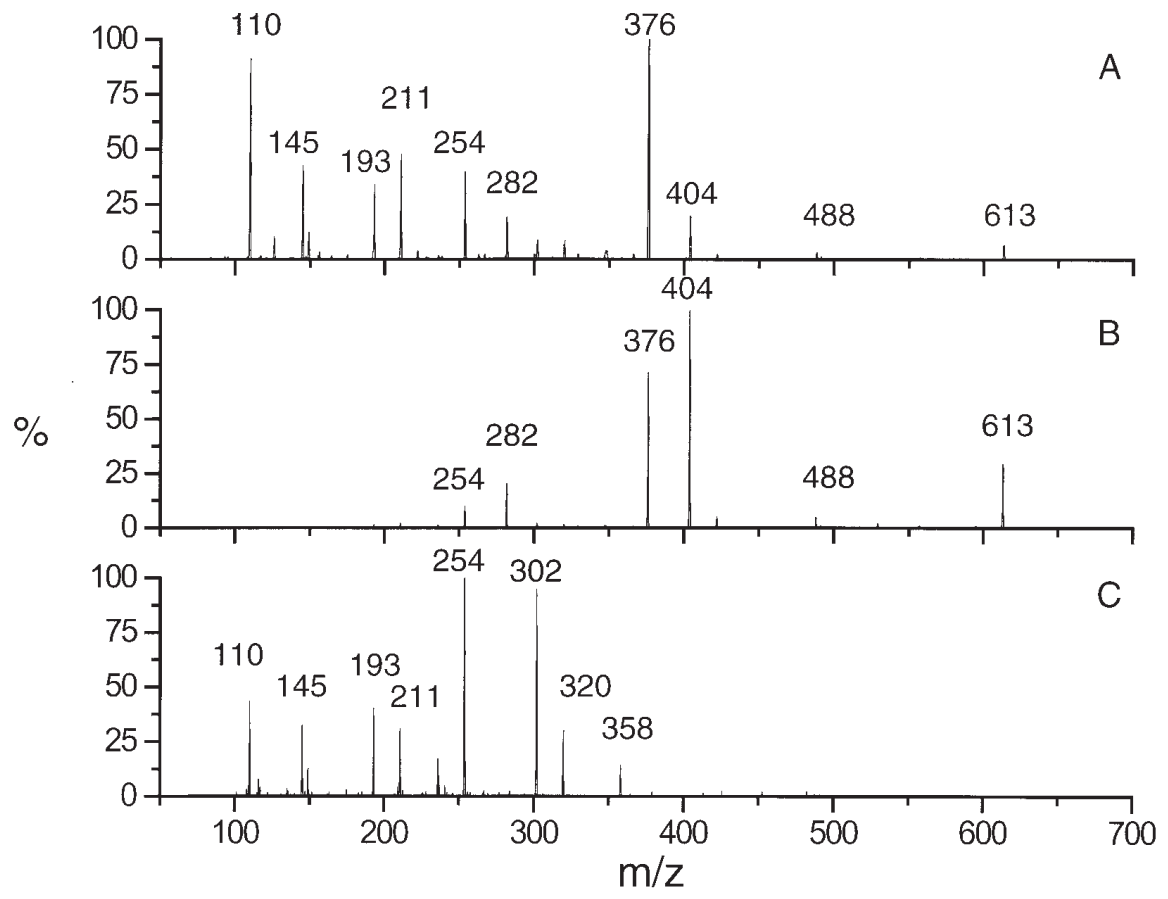

Figure 3. CID spectra of remikiren A) triple quadrupole instrument at $50 \mathrm{eV}$ B) LCQ MS 631.4 -> spectra at $60 \%$ C) LCQ MS ${ }^{3} 631.4$-> 376 spectra at 90 and $60 \%$.

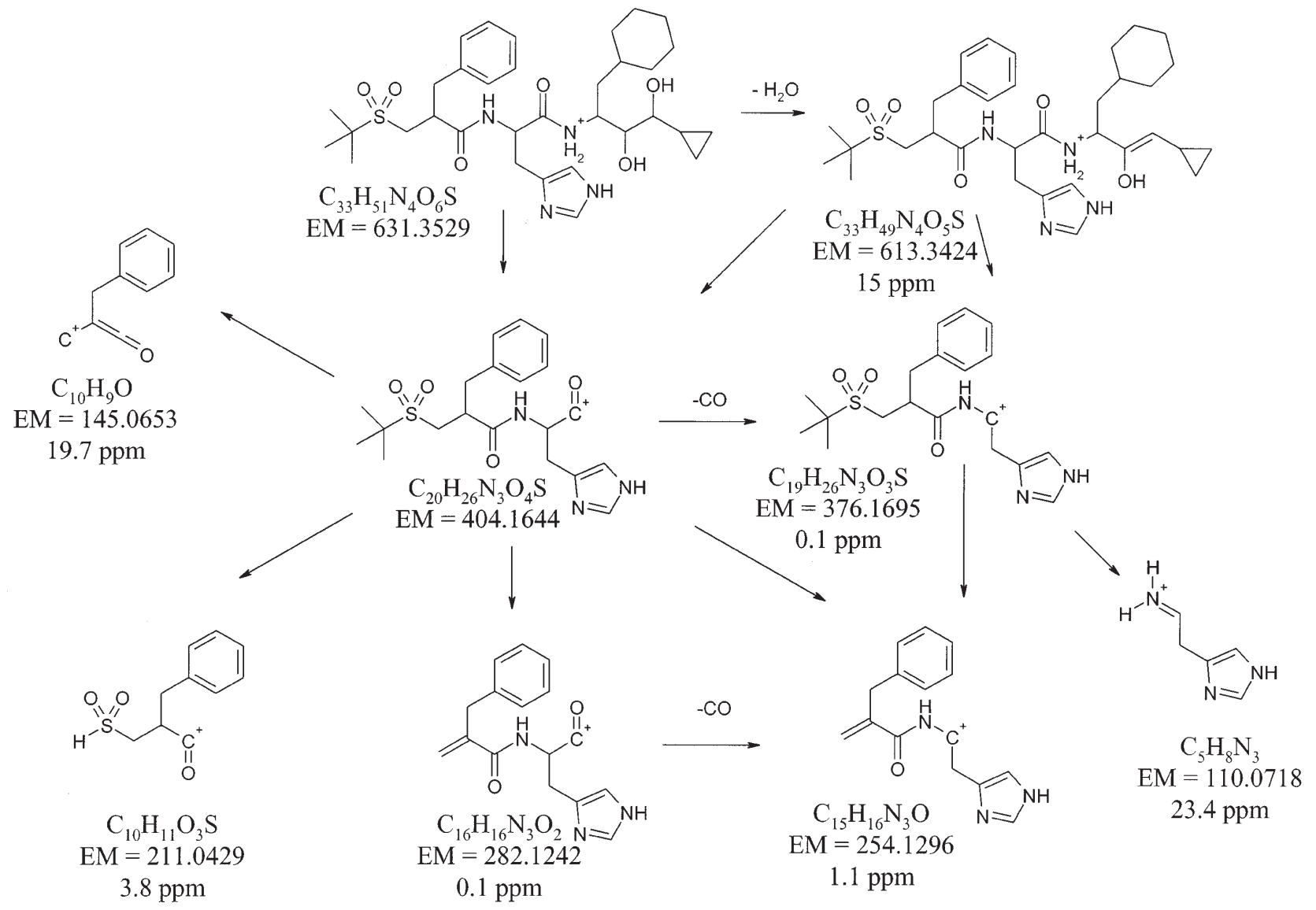

Scheme 2. Fragmentation pathway of Remikiren. 


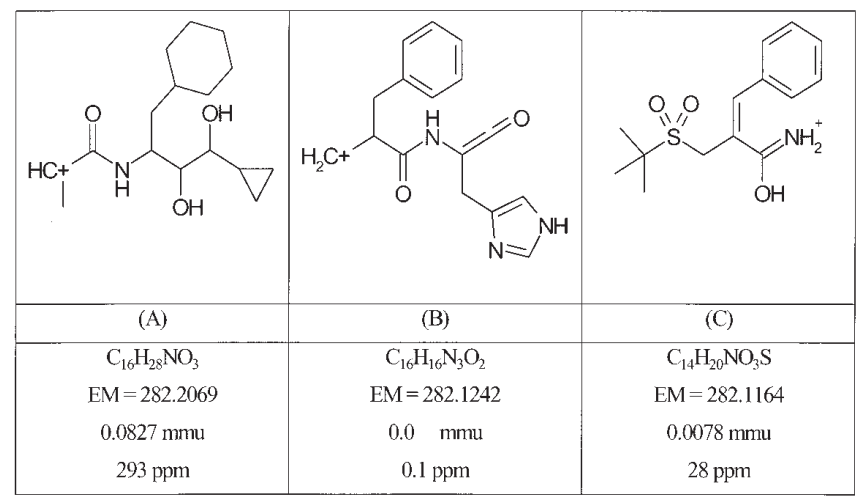

Scheme 3. Postulated structures of the fragment at $m / z 282$.

at $m / z$ 282. Therefore, we can exclude that the fragment at $\mathrm{m} / \mathrm{z} 282$ has the structure A (Scheme 3). This shows the limitations of triple quadrupole and ion trap approaches and one would think based on this experiment that structure B is the most probable one. However, this conclusion is not supported by strong data. The product ion spectrum obtained on a QqTOF instrument is illustrated in figure 4A. In this case a single lock mass calibration was performed for the ion at $\mathrm{m} / \mathrm{z}$ 404. With the constraint of the parent drug only 3 elemental compositions are possible within 5 ppm mass accuracy: i) $\mathrm{C}_{10} \mathrm{H}_{22} \mathrm{~N}_{2} \mathrm{O}_{5} \mathrm{~S}, \mathrm{EM}=282.1249$ ii) $\mathrm{C}_{16} \mathrm{H}_{16} \mathrm{~N}_{3} \mathrm{O}_{2}, \mathrm{EM}=$ 282.1242 , iii) $\mathrm{C}_{18} \mathrm{H}_{28} \mathrm{O}_{3} \mathrm{EM}=282.1255$. Based on the structure on the molecule it is obvious that only $\mathrm{C}_{16} \mathrm{H}_{16} \mathrm{~N}_{3} \mathrm{O}_{2}$ makes sense, which is in full agreement with structure $\mathrm{B}$ in scheme 3. The resolution of the peak at $\mathrm{m} / \mathrm{z} 282$ is approximately 5000 at FWHM. For a given molecule, different fragmentation pathways may generate isobaric fragments with different elemental compositions. For example if fragment $(\mathrm{C})$ in scheme 3 is fragment of remikiren and is present at the same intensity as fragment (B), resolution of the two fragments would not be possible and the mass inaccuracy would be much larger. The peak would also be slightly broader. Therefore, with medium resolution accurate mass measurements, the possibility of overlapping fragments should be carefully considered when proposing a fragmentation mechanism.

Another interesting fragment is the ion at $\mathrm{m} / \mathrm{z}$ 211. We know that this fragment is also generated from fragment $\mathrm{m} / \mathrm{z} 376$ and again several structures can be postulated from our experience or proposed by the Mass Frontier software. However, none of them is compatible with the accurate mass measurement (211.0437) of this fragment. Within $10 \mathrm{ppm}$ only three elemental compositions are possible i) $\mathrm{C}_{10} \mathrm{H}_{11} \mathrm{O}_{3} \mathrm{~S}$ $\mathrm{EM}=211.0429$ ii) $\mathrm{C}_{16} \mathrm{H}_{5} \mathrm{~N} \quad \mathrm{EM}=211.0422$ iii) $\mathrm{C}_{13} \mathrm{H}_{9} \mathrm{NS}$ $\mathrm{EM}=211.0456$. Only elemental composition i) is compatible with the structure of the molecule (Scheme 3) corresponding to a neutral dimethylvinyl elimination at the sulfonyl moiety, which is quite atypical. Based on accurate mass measurements and $\mathrm{MS}^{\mathrm{n}}$ experiments, the fragmentation pathway of remikiren could be established as illustrated in scheme 3. With more sophisticated software tools most of the fragments present in the spectrum could be identified automatically. This would be very useful for high throughput analyses. In the case of remikiren, the precursor ion $[\mathrm{M}+\mathrm{H}]^{+}$could not be used for lock mass calibration and the fragment due to the loss of water was found to be less suitable for accurate mass measurements in the low mass range. It is also interesting to mention that in the low mass end we obtained accuracies in the $20 \mathrm{ppm}$ range. Using the information gained from the drug candidate, metabolites can be characterised rapidly and with high confidence. The CID spectra of three metabolites of remikiren are illustrated in figure $4 \mathrm{~B}-4 \mathrm{C}$ and are in full agreement with the proposed fragmentation pathway.

One other task in drug metabolic investigations is the rapid screening of metabolites in the first human studies. In general, metabolite concentrations are much lower in vivo than in vitro samples, which analytically is more challenging. One efficient way is to use DDE experiments as illustrated previously. On QqTOF and ion trap instruments only product-dependent scans are possible. By this way it is possible to confirm the presence of known metabolites but it is not efficient to identify unknowns. There is a physical
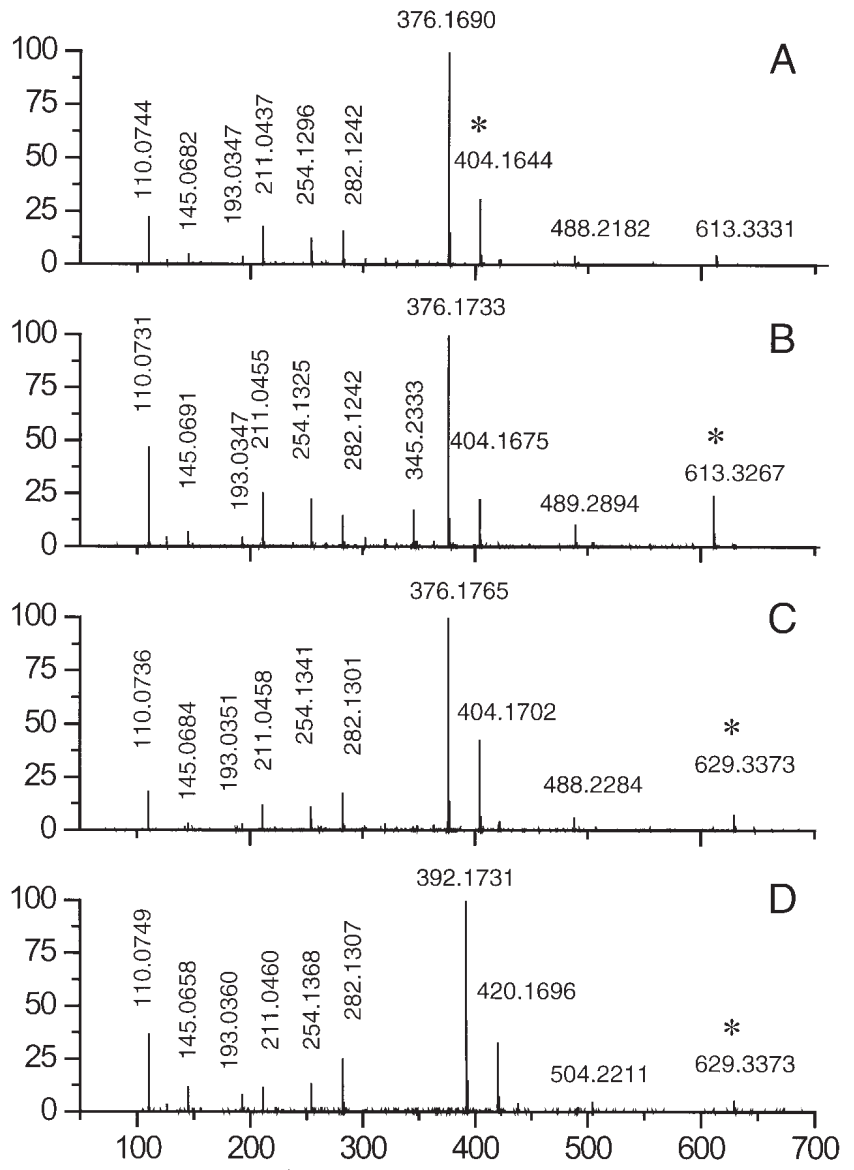

Figure 4. QqTOF CID spectra at $35 \mathrm{eV}$ of A) remikiren (II) B) oxidative metabolite (III) C) hydroxy metabolite (IV) D) hydroxy metabolite (V) * Lock mass. 

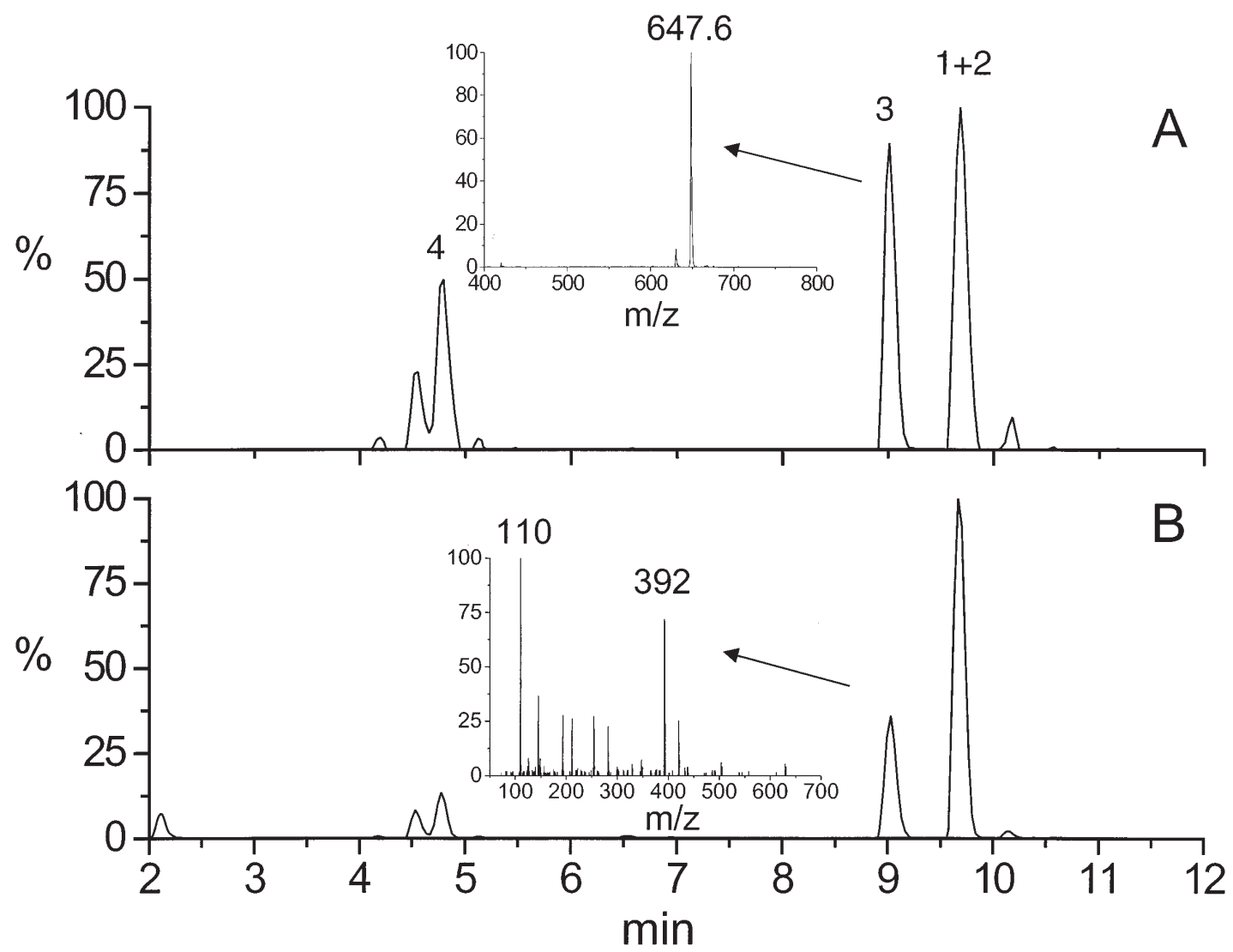

Figure 5. LC-MS/MS DDE experiment on a triple quadrupole of remikiren and three of its metabolites A) precursor experiment $m / z 110$ (survey scan) B) TIC trace of the product ion (dependant scan) 1 compound (II), 2 compound (III), 3 compound (IV), 3 compound V.

limitation to set-up the experiment in order to cover all possibilities. Triple quadrupole instruments have a unique scan mode called the precursor scan mode, where Q1 is set in scan mode while Q3 is set at the mass of a representative fragment. By selecting an appropriate precursor ion it is possible to find almost all compound related metabolites that are ionised under theses conditions and not suppressed by the matrix in the total ion current trace. Ideally, this fragment should be present is all metabolites and in the part of the molecule that is not metabolised. This approach is illustrated in figure 5. In this case the fragment at $\mathrm{m} / \mathrm{z} 110$ was selected as the precursor ion. Due to the high selectivity of the precursor scan mode, acting as MS filter, metabolites can be identified in the TIC trace of the LC-MS run very easily as shown in figure $5 \mathrm{~A}$. In the full scan mode the metabolites would be hidden under the background and one would have to search them.

\section{Conclusion}

The utility of accurate mass measurements of product ion fragments with QqTOF at medium resolution, in association with MS interpretation software and ion trap mass spectrometry for the elucidation of the fragmentation pathways was illustrated on moclobemide and remikiren. The use of more sophisticated software would allow the automated interpretation of mass spectra. This would be very useful for high throughput analyses. Typically, mass accuracies better then $10 \mathrm{ppm}$ were obtained, which are not sufficient to define a unique composition for a fragment. The combination of QqTOF and $\mathrm{MS}^{\mathrm{n}}$ data overcomes partly this limitation. Even in cases where these values exceed $10 \mathrm{ppm}$ a structure allocation would be possible. Therefore, a systematic investigation of CID fragmentation of small molecules using accurate mass QqTOF is very valuable to gain knowledge on these mechanisms. The understanding of these mechanisms is the key to the set-up of appropriate DDE experiments for fast and automated screening of metabolites in in vitro or in vivo samples.

Ideally, for the investigations of the biotransformation of drug candidates by LC-MS one would like to have a mass spectrometer, which provides fast scanning, accurate mass at medium resolution, $\mathrm{MS}^{\mathrm{n}}$, and precursor ion scanning $\left((\mathrm{QqQ})^{\mathrm{n}} \mathrm{TOF}\right)$. Unfortunately, this is still a dream. However, this study shows that the structure elucidation and screening 
of metabolites at low concentration by mass spectrometry requires orthogonal approches.

\section{Acknowledgement}

We would like to thank R. Gasser for performing the experiments involving rat hepatocytes and $H$. Dollt and W. Meister for fruitful discussions.

\section{References}

1. Lee, M. S.; Kerns, E. H. Mass Spectrom. Rev. 1999, 18, $187-$ 279.

2. Niessen, W. M. A. J. Chromatogr. A 1998, 794, 407-435.

3. Bruins, A. P. Trends Anal. Chem. 1994, 13, 37-43.

4. Busch, K.; Glish, G.; McLuckey, S. Mass Spectrometry/Mass Spectrometry: Techniques and Applications of Tandem Mass Spectrometry; Weinheim: VCH, 1988.

5. Fernandez-Metzler, C. L.; Owens, K. G.; Baillie, T. A.; King, R. C. Drug Metab. Dispos. 1999, 27, 32-40.

6. Hopfgartner, G.; Vetter, W.; Meister, W.; Ramuz, H. J. Mass Spectrom. 1996, 31, 69-76.

7. Hopfgartner, G.; Chernushevich, I. V.; Covey, T.; Plomley, J. B.; Bonner, R. J. Am. Soc. Mass Spectrom. 1999, 10, 13051314.
8. Morris, H. R.; Paxton, T.; Dell, A.; Langhorne, J.; Berg, M.; Bordoli, R. S.; Hoyes, J.; Bateman, R. H. Rapid Commun. Mass Spectrom. 1996, 10, 889-96.

9. Shevchenko, A.; Chernushevich, I.; Ens, W.; Standing, K. G.; Thomson, B.; Wilm, M.; Mann, M. Rapid Commun. Mass Spectrom. 1997, 11, 1015-24.

10. Yang, M.; Bonner, R.; Burton, L.; Bloomfield, N.; Le Blanc, Y. Presented at the 48rd ASMS Conference on Mass Spectrometry and Allied topics, Long Beach, CA, June 11-15, 2000.

11. Guilhaus, M.; Selby, D.; Mlynski, V. Mass Spectrom. Rev. 2000, 19, 65-107.

12. Zhang, H.; Henion, J.; Yang, Y.; Spooner, N. Anal. Chem. 2000, 72(14), 3342-2248.

13. Tyler, A. N.; Clayton, E.; Green, B. N. Anal. Chem. 1996, 68(20), 3561-3569.

14. Wu, Q. Q. Anal. Chem. 1998, 865-872.

15. Eckers, C.; Wolff, J.-C.; Haskins, J.N.; Sage A.B.; Bateman, K.G. Anal. Chem. 2000, 72(16), 3683-3688.

16. Jauch, R.; Griesser, E.; Oesterhelt, G.; Arnold, W.; Meister, W.; Ziegler, W.; Guentert, T. Acta Psychiatr. Scand. Suppl. 1990, 87-90.

17. Jauch, R.; Schmid, P.; Fischli, W.; Meister, W.; Maurer, R.; Wendt, G. Xenobiotica 1996, 26(3), 285-95. 